\title{
SOME OBSTRUCTIONS TO SPEECH-DEVELOPMENT.*
}

\author{
BY G. IIUDSON-MAKUEN, M. D., PIIILADELPHIA.
}

The tendency toward the development of speech seems to be an inherited one, and when even in the first year a child fails to exhibit indications of this tendency, we may be sure that there is something wrong. It is not always easy, however, to determine just what the difficulty may be, and it is of the various possible reasons for delayed speech-development that I wish to speak to you, adding a word of suggestion, perhaps, as to the best methods for removing them.

Speech has been defined as "a system of articulate words adopted by convention to represent outwardly the internal processes of thinking"; but speech does more than this, for it not only represents the internal processes of thinking, but it is so closely interwoven with them as to be absolutely essential to their development. Speech is also one of the essentials to the highest physical deevlopment, for its use tends to strengthen some of the most important muscles of the body, to expand the lungs and aerate the blood. Deprive a child of speech, therefore, and you deprive him of one of the most important tools of the mind, as well as one of the most important exercises of the body.

The faculty of speech is presided over by delicate and complicated cerebral areas inciting to action, and working in harmony with, peripheral organs having other important bodily functions in addition to those of voice-and speech-production. Defective speech, therefore, having its origin in a defective action of some of these important central areas and peripheral organs, is a serious malady and deserves most careful consideration.

In order to have a clearer understanding of the subject, and at the risk of being too elementary in my talk to yotr, allow me to refer briefly to the various mechanisms of speech.

For purposes of study, the mechanisms of speech may be divided into two general classes, the central and the peripheral mechanisms. The peripheral mechanisms are three in number, namely, the respiratory, phonatory, and articulatory mechanisms. The respiratory mechanism, with its large muscular attachments, furnishes the motor power for speech, which, together with the phonatory mechanism, produces the material of which speech is made, namely,

*An address delivered before the Teachers' Association of the Pennsylvanla School for the Deaf, at Mt. Alry, Philadelphia, Pa. 
voice. The articulatory mechanism not only modifies the character of voice, but it also molds and articulates it into the symbols, which in their different combinations represent intelligible speech.

The three peripheral mechanisms constitute what may be called the physics or mechanics of speech, and their normal action and development depend, first, upon their structural adequacy, and second, upon their control by the higher central mechanisms situated in the brain and spinal cord.

Among the structural conditions of the peripheral mechanisms that may interfere with the development of speech in children may be mentioned, first, deformities of the thorax, interfering with respiration and breath-control in general; second, deformities with tumors of the larynx, interfering with the production of voice, which is the essential element of speech, and third, the numerous irregularities of structure, and pathologic conditions in the month, pharynx, nose, and ear.

Deformities of the palate, including the alveolar arches and teeth, are frequent causes of faulty speech-development in children. The speech resulting from a cleft palate is characteristic, and it is well known because of its pronounced and conspicuous nature; but the lesser deformities of the palate and irregularities of the teeth, while equally as pósitive, are not as easily recognized, because their results are manifest only to the trained ear. As is well known, the cleft, or so-called hare-lip, also frequently accompanies deformities of the palate, adding materially to the difficulties of normal speech-development.

Deformities of the tongue have long been recognized as obstructions to speech-development, but the importance of some of them, I think, has been overestimated. The large, unwieldy tongue of the cretinoid child and of the Mongolian type of imbecility and idiocy is well known, and it is undoubtedly to some extent a cause of the defective speech in these unfortunate individuals; but it is probably quite as much a result of their lack of speech-development as a cause. The large tongue, however, is not always a mark of imbecility or even of feeble-mindedness, for it is sometimes found in an otherwise normal child, and the scientific name for it is macroglossia.

A short lingulal frenum, or tongue-tie, has been and is now regarcled by many as being the chief cause of the lack of speechdevelopment in young children; but from my own experience I conclude that a short lingual frenum has but little effect upon the character of the speech. It does, to be sure, tend to bind the tip of the 
tongue down in the floor of the mouth, and to limit its action; but is it not a fact that more disorders of speech result from too great activity of the tongue than from too little? In order to limit the activities of the tongue, many teachers now advocate the holding of it clown against the lower teeth cluring the emission of the labiodental and palatal sounds. Notwithstanding this fact, however, it is quite possible that a short lingual frenum may so hamper the normal action of the tongue in young infants as to make it figure to some considerable extent in the causation of delayed speechdevelopment.

Mechanical obstructions to the development of speech may be looked for also in the pharynx, and among these enlarged tonsils and adenoids are the most frequent. Moreover, these abnormal pharyngeal growths interfere with the development of speech psychically as well as physically, and therefore they assume a twofold importance. I am inclined to think that the psychic influences brought about by the respiratory and circulatory disturbances which result from enlarged and diseased tonsils and adenoids are greater, so far as speech. is concerned, than the mere mechanical obstructions coming from these sources. The general health, also, of the vitherwise not-too-strong child may be so greatly impaired by these conditions as to render him incapable of psychic development, and therefore the speech naturally must suffer in consequence.

Intra-nasal obstructions also tend to impede normal speechdevelopment in a somewhat similar manner, depriving the child of sufficient oxygen and interfering with the normal development of the general respiratory system. No one of the mechanisms of speech can escape the deleterious influences of faulty nasal breathing. The respiratory mechanism suffers by a lack of development and by the more or less constant congestion and resultant catarrh of the mucous membrane, which always accompanies this condition. Rhinitis, pharyngitis, laryngitis, and bronchitis are common in such cases, and these conditions render speech difficult and in some instances quite impossible to the average individual. Moreover, the ill effects of intra-nasal obstructions and intra-nasal pressure upon speech and voice are progressive in their character and increase with the age of the patient. This is more especially true when the trouble arises from deformities of the septum rather than from hypertrophies of the turbinate bones, because the hypertrophies tend to atrophy in later life, and thus nature comes to the relief. The nasal operation, therefore, most frequently indicated in adult life is generally one for the correction of a deformity of the sep- 
tum; and, unfortunately, the older the patient grows the greater the discomfort and the greater the injury to the speech from such a condition. This, I suppose, is in accordance with the principle that the vigor of youth enables one to overlook and overcome conditions which become a real annoyance and even an obstruction to progress in later life.

It is not alone the obstruction to breathing which arises from these nasal deformities that is prejudicial to normal speech-development; but the intra-nasal pressure which often accompanies them is a factor of almost equal importance.

I have been impressed with the large number of stammerers, for instance, who have intra-nasal pressure, and whose speech-defect, I am convinced, is greatly aggravated by this condition. If we look upon stammering as a neurosis-and I think that it may be so regarded in many instances-we may not be going far amiss in attributing its origin to a reflex distubance arising from pressure within the nose.

We come now to the most important of all obstructions to speechdevelopment, namely, disorders of the ear and the resultant hardness of hearing and deafness. If we keep in mind the fact that the ear is an integral part of the respiratory tract, being merely an extension of the naso-pharynx, with highly specialized functions, we shall be in a position to understand some of the conditions of this organ which tend to disturb its two important functions, name$1 y$, the function of hearing and the function of equilibrium, the former of which, of course, will especially concern us at this time.

The ear, as I have said, being an extension outward and upward on either side of the naso-pharynx, is subject itself to the various inflammatory conditions common to this region, and it partakes oftentimes of the results of the diseases of those portions of the upper respiratory tract which are nearest to it, namely, the nose and pharynx. This explains why intra-nasal obstruction and pressure and hypertrophied tonsils and adenoids so often affect the ear, and why they become the chief causal factor in the various diseases of this organ.

Earache, for instance, a condition which depends upon an acute inflammation of the middle portion of the ear, is almost always due to so-called colds in the'head, which are in turn the direct results of nasal obstruction and hypertrophied tonsils and adenoids. These acute attacks, if not carefully treated, result, of course, in chronic inflammation and discharge from the ear, with mastoid complications and more or less destruction to the aural mechanisms, whose 
function it is to conduct the sound-waves to the inner ear and thence through the auditory nerve to the centers of hearing in the brain.

My whole hour, of course, would scarcely suffice accurately to describe to you the clelicate and beatiful structures which combine to form the mechanisms of the ear, and the various diseases which attack them. I have described briefly to you the peripheral organs of speech and I have referred to the chief disorders of them which result in obstructions to speech-clevelopment, and it now-remains to speak of the central mechanisms of speech and some of the causes which lead to their faulty development.

The central mechanisms of speech are four in number, namely, the auditory, the visual, the glosso-kinesthetic, and the chiro-kinesthetic centers, or the centers for hearing, seeing, speaking, and writing words. It seems to me that it would simplify our study of the cerebral mechanisms of speech to add still another center, namely, the tactile center, although this could scarcely be so definitely localized as the others, because the tactile speech-centers are more widely distributed throughout the cerebral cortex.

The atclitory speech-center is merely a differentiated or specialized portion of the so-called general auditory ceinter. It is the particular point at which word images are registered in the brain, to be afterward revived or recalled for purposes of voluntary speech. Hearing, therefore, is essential to the natural development of the auditory speech-center. The untaught deaf child has no development of the auditory speech-center. He has no registration of word-imnges, and therefore he cannot learn to speak in the usual mannei. The partially deaf child, on the other hand, has distorted word-images, because he hears words inaccurately, some of the important elements which enter into their formation escaping him entirely. Moreover, the accurate formation of word-images in the auditory center of the brain depends in great measure upon the child's ability accurately to phonate and articulate words, because the formation and registration of word-images take place simultaneously with the development of the words in speaking. It may be said, therefore, that the child must speak the word before the formation of the image in the auditory center can be completed, and if for any reason, peripheral or otherwise, the word is inaccurately spoken, it will gradually come to be inaccurately registered and the image will be a distorted one.

It is this fact that prompted me some time ago to write a paper entitled "Do we hear ourselves as others hear us?" which question 
I answered in the negative. I tried to show that we do not hear ourselves as others hear us, but each of us has individual standards of speech which differ from those of all others, and our own speech is as near as we can make it an exemplification of our own standards. No one has ever spoken better than he knows, and for one to improve his speech, he must elevate his standards of speech.

The totally deaf child, as I have said, develops no auditory wordimages, and the partially deaf child develops distorted word-images. Now I wish to emphasize the fact that a child may have normal hearing-power and yet, owing to some obstructions in the other peripheral or central mechanisms of speech, he may, like the totally deaf child, develop either no word-images at all, or, like the partially deaf child, he may develop distorted ones. In other words, the development of word-images depends in large measure upon the child's mental and physical ability to produce words.

I have described the various conditions of the peripheral organs which may lead to faulty speech-development and to distorted wordimages, and it remains now to speak of the cerebral conditions which may have precisely the same effects.

When a person speaks spontaneously, the cerebral processes are somewhat as follows: (I) Ideas are aroused; (2) from the mental operations conducted with the help of these ideas a resultant product issues; (3) the product is clothed in words; (4) the central innervations necessary for their externalization are brought about; and finally (5), these innervations are conducted to the peripheral organs of speech in their proper order and intensity. Spontaneous speech, therefore, requires for its production more than the mere storage of word-images in the auditory word-center. It requires, first of all, the arousing of ideas and the issuance of a resultant product from certain mental operations conducted with the help of these ideas.

The mentally deficient child, therefore, cannot speak accurately or connectedly, because he has no well-defined ideas with which to conduct the mental operations necessary to the formation of the resultant product. He has no resultant product, even if he had words with which to clothe it. Again, other subnormal children may be mentally alert; that is to say, they may be able to arouse ideas and conduct with them the mental operations necessary for the issuance of a resultant product, and they may also have a good supply of word-images in their auditory word-centers with which to clothe their resultant product, yet they may be entirely unable to arouse the innervations which are necessary for the externalization of 
words; or, having aroused the necessary central innervations, they may be unable to conduct these innervations to the peripheral organs of speech in their proper order and intensity.

This brings us to a study of the glosso-kinesthetic center, or the so-called Broca's motor center of speech. As the term kinesthetic suggests, we have stored in this center memories of the movements of the peripheral muscles in the production of speech. The child who does not hear speech, as I have said, will have no differentiated auditory word-center, and therefore he cannot, of his own accord, learn to speak. Moreover, the child who has not learned to speak can have no glosso-kinesthetic speech-center or memories of the muscular movements necessary for the development of speech. The child having defective speech, on the other hand, will not only have distorted auditory word-images, but he will also have defective kinesthetic memories of the muscle-movements necessary for the production of words, and the correction of his defective speech consists in the correction of his defective kinesthetic word-memories and through them the correction of his distorted auditory wordimages.

The auditory center and the glosso-kinesthetic center are the two centers chiefly employed in speech, and together they have been called the primary speech couplet. The centers employed in reading and writing are the visual and the chiro-kinesthetic centers, and as reading and writing are closely allied to hearing and speaking, this secondary couplet is also connected with the primary couplet by so-called commissural fibers: This explains why it is that in normal hearing-children, the eye may be used to some extent as well as the ear in the development of speech, and the act of writing is generally acquired after the child has learned to speak and read. Writing, however, being accomplished by the larger muscles of the hand and arm, is a voluntary act, and, unlike the clevelopment of speech in the hearing-child, it is acquired by long and painstaking voluntary effort.

In the normal development of speech, as we have seen, the child, after hearing words, attempts to imitate them, and he checks up the results of his efforts by drawing a mental comparison between his own subjective hearing of them and his original objective hearing of them. In this manner he finally forms his individual standards of speech. These standards, as I have said, differ in different in. dividuals; but each one's own standards are generally quite satisfactory to himself.

Let us consider now what takes place in the brain of the totally deaf child when we teach him to speak. We may suppose that 
many deaf children, like hearing children, are possessed of an inherited tendency to speak, and this fact, of course, greatly facilitates our work with them. Indeed, I have no doubt but that if deafness could be detected in children as carly as the beginning of the second year, and if training in speech-and lip-reading conld be systematically and vigorously begun and carried on at that time, we could get much more satisfactory results than according to our present methods. It is in the second year that the inherited tendencies toward speech-development are most pronounced, and it is cluring babyhood that perhaps the greatest attention is given to the development of these tendencies in hearing children; but as soon as it is found that children are deaf, we all begin immediately to neglect them and in many other ways to spoil them. If my own child were deaf, I think I would talk to it and have it talked to all the more because of its deafness, and thus even in the first year try to have it learn lip-reading and to have it continue to develop voice and speech from the small beginnings which even deaf children make in the way of crying, babbling, prattling, etc.

When we teach the deaf to speak, as I have said, we are handicapped by the absence of any development whatsoever of the auditory speech-center. The child has no hearing for speech, either subjective or objective, and we must therefore substitute some other sense or senses for that of hearing. I have explained to you how even in hearing children the visual sense nay be used to some extent in the development of speech, and you know that the tactile sense is also made of great service. In teaching the deaf child to speak, the tactile sense is of the first importance, for it must take the place which the hearing sense occupies in normal children. I would substitute, therefore, for the auditory center in our diagram the tactile conter, and this would give us a scheme for studying the cerebral operations in the development of speech in the deaf.

SUMMARY.

Human speech has been called the greatest of miracles and the wonder of creation. "In the beginning was the WWord, and the Word was with God and the'Word was God." Even before thought and reasoning were possible there probably existed the word, and man thus became exalted above the brute creation and was made only a little lower than the angels. A study, therefore, of the obstructions to the development of speech and methods for their removal would seem to be worthy of our best efforts.

The chief obstruction to the normal development of speech, of conrse, is deafness, because hearing is absolutely essential to the 
natural and automatic development of the cortical speech-centers. The functions of the ear, therefore, should be carefully guarded, and every possible means should be employed for their development, because even a little hearing is better than no hearing at all.

Deformities of the mouth, pharynx, and nose, as well as those of the larynx and the respiratory organs, are in themselves prejudicial to normal speech-development, and they are frequently the direct cause of the various diseases of the ear which result in hardness of hearing and oftentimes.in deafness. Deafness, as I have stated, is the chief obstruction to speecli-clevelopment, because it: interferes with the normal cerebral development, and speech itself is more cerebral, or cortical, than peripheral. That is to say, if the speech-centers in the brain are normal in their development and action, fairly good speech may be produced with very imperfect peripheral mechanisms. Intelligible speech has existed, for instance, after the complete removal of the tongue and even of the larynx, and a cleft palate affects the speech only to a certain limited degree.

So much is speech a product of the brain that the articulatory muscles have been called the mental muscles. Good speech cannot issue from a brain diseased, but it is the result of the fullest development of the higfier intellectual centers of the brain. The lower speech-centers which I have described can work effectively in the externalization of speech only when they are controlled by other and higher centers situated in the fore-brain. Feeble-mindedness, therefore, imbecility, and idiocy are incompatible with gcod speech-development, because without normal intellectuality and mentality there can be no normal development of the lower centers and peripheral organs of speech.

All speech-training, therefore, whether the defects be on account of cleafness or on account of the thousand and one other things that may impede its development, must be carried on simultaneously with psychical training. Speech-training consists, after all, only in an effort to help the individual by means of the development of the higher intellectual centers of his brain himself to control and train his lower speech-centers, and thus through them the peripheral mechanisms of speech. All children should have a certain amount of speech-training, and thus not only would the expression of thought be placed upon a higher plane, but that portion of the brain which manufactures thought would be itself more highly developed.

ı́27 Walnut Street. 